\title{
The Use of APACHE II Scoring System for Predicting Clinical Outcome of Patients Admitted to the Intensive Care Unit: A Report From a Resource-Limited Center
}

\author{
Maryam Farajzadeh ${ }^{1}$, Elham Nasrollahi ${ }^{1}$, Yaser Bahramvand ${ }^{1}$, Vahid Mohammadkarimi $\mathbb{i}^{2}$, Behnam \\ Dalfardi ${ }^{3}$ and Amir Anushiravani ${ }^{4, *}$ \\ ${ }^{1}$ Student Research Committee, Shiraz University of Medical Sciences, Shiraz, Iran \\ ${ }^{2}$ Department of Internal Medicine, Shiraz University of Medical Sciences, Shiraz, Iran \\ ${ }^{3}$ Endocrinology and Metabolism Research Center, Institute of Basic and Clinical Physiology Sciences, Kerman University of Medical Sciences, Kerman, Iran \\ ${ }^{4}$ Digestive Diseases Research Institute, Tehran University of Medical Sciences, Tehran, Iran \\ "Corresponding author: Digestive Diseases Research Institute, Tehran University of Medical Sciences, Jalal Al Ahmad Blv, Tehran, Iran. Tel: +98-9173160616, Email: \\ amiranush23@yahoo.com
}

Received 2020 March 20; Revised 2020 October 31; Accepted 2020 November 18.

Keywords: Intensive Care Units, Prognosis, APACHE

\section{Dear editor,}

Scoring systems help predict the duration of hospital stay and outcome of critically ill patients. The APACHE score is possibly the best-known and most widely used scale for evaluating the severity of acute illness (1). The APACHE scoring system was introduced in 1981 by the Medical Center of George Washington University as a method for measuring the severity of the disease (2). This system was edited by Knaus et al. and named APACHE II $(3,4)$. This scoring system may also be helpful in patient selection for admission to the Intensive Care Units (ICUs), particularly when the number of ICU beds is limited. Our study was done to emphasize the use of these scores by assessing patients' outcomes in ICUs of a resource-limited region in Iran using the APACHE II scale.

A cross-sectional study was conducted in 2015 and 2016. All cases over 16 years of age with a hospital duration stay of more than eight hours were included. Burn patients or those who were brain-dead were excluded. In the case of multiple ICU admissions, data of the first admission were recorded. Demographic data, including patients' age, sex, length of admission, outcome, and final diagnosis, were recorded using pre-designed data collection forms. A twopart checklist was used for data gathering. The first part included the demographic information, and the second part included the standard APACHE II checklist. In this method, numerical values that were assigned to each variable were summed up and reported for each patient. The mean, standard deviation (SD), minimum, and maximum, as well as the sensitivity, specificity, and positive and negative predictive values were reported, and finally, a ROC curve was drawn. A one-sample $t$-test was used for comparing the mean scores with fixed numbers, and the independent $t$ test was used for comparing the mean scores between the two independent groups (discharged cases versus expired patients). The Kolmogorov-Smirnov test was used for examining the normalization of quantitative data in each group, and the chi-square test was applied for determining the relationship between the two classified qualitative factors. We used SPSS ver.18 and MedCalc software to analyze the data.

The APACHE II score was used to evaluate the severity of disease among the included patients. This scoring system is composed of three main components: (1) Acute physiology scores, (2) age points, and (3) chronic health points.

A total of 185 patients met the inclusion criteria and were included, of whom $68.1 \%$ were men (126 patients), and $31.9 \%$ were women (59 patients). The mean age \pm SD for the total population was $45.56 \pm 23.03$ years $(42.74 \pm 23.30$ for men and $51.59 \pm 24.62$ for women). Also, 67 (36\%) patients were admitted to the medical ICU and 118 (63.8\%) cases to the surgical ICU. The mean duration of hospital stay was 8.8 \pm 7.05 days.

The independent $t$-test was used for comparing the 
mean age among discharged $(n=103)$ and expired $(n=82)$ patients, as well as for comparing the duration of hospitalization. The mean age of expired patients was $54.45 \pm 22.25$ years, and the mean age of discharged patients was $38.49 \pm$ 23.11 years. A chi-square test was also used to investigate the relationship between patients' gender and their outcome. According to our results, there was a significant increase in the patients' mortality with advancing age $(\mathrm{P}<0.001)$.

The sensitivity, specificity, and positive and negative predictive values for different cutoff points are reported in Table 1 using MedCalc software. As can be seen, the best cutoff point was determined to be 19 .

This APACHE cutoff was highly specific to our patients. Figure 1 displays the ROC curve. The area below the ROC curve was 0.888 (standard error $=0.026$ ).

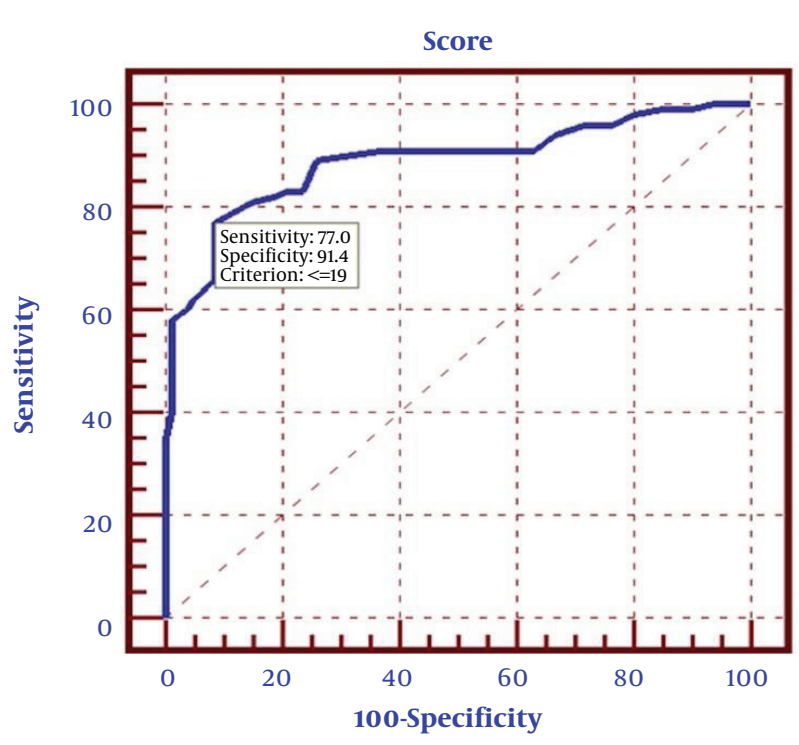

Figure 1. The APACHE II ROC curve to predict hospital mortality

The mean APACHE II score was $14.02 \pm 8.48$ for the discharged patients, which was significantly lower than the reference point (score of 20$)(\mathrm{P}<0.001)$. This value was $27.46 \pm 6.61$ for the expired cases, which showed a significant difference from the aforementioned cutoff $(\mathrm{P}<$ 0.001).

In this study, the mean age of the included subjects was different between the two groups (expired and discharged) and was significantly higher among expired patients. Our results were consistent with the results by Niewinski et al. (5). In contrast, Cho et al. (6) believed that age did not have a significant impact on the mortality of ICU-admitted patients. The mean duration of hospitalization was not significantly different between both studied groups. The results of our research were consistent with the findings of studies by Giangiuliani et al. (7) and Donahoe et al. (8). There was no significant difference between both studied groups regarding the gender ratio. The findings by Ho et al. support the results of our study (9). We measured the APACHE II score for discharged patients, and a mean value of 14 was obtained. Comparing this value with a reference point of 20 showed that the mean APACHE II score in discharged patients was significantly smaller than 20. Also, by measuring the APACHE II score among expired patients, a mean score of 27 was found, and comparing it with a score of 20 showed that the mean APACHE II score in expired patients was significantly higher than 20. Our results were consistent with studies by Cho et al. (6), Giangiuliani et al. (7), and Ho et al. (9). According to Anushiravani et al. (10), non-office hour admission did not affect the mortality of patients admitted to an internal medicine ICU; however, they showed that the highest peak of mortality was between 10 PM and 2 AM. The other important point is that fatigue can surely be a cause of mismanagement in any medical professional (10). We showed that patients who had a lower APACHE II score would have a better prognosis. A cutoff point of 19 can be used for selecting patients to be admitted to the ICU in our hospital. In addition, more studies are required to evaluate the results of the implementation of this APACHE admission cutoff point.

\section{Footnotes}

Authors' Contribution: Maryam Farajzadeh, Elham Nasrollahi, Yaser Bahramvand, Vahid Mohammadkarimi, Behnam Dalfardi, and Amir Anushiravani contributed to the conception of the study and its design. Maryam Farajzadeh, Yaser Bahramvand, and Elham Nasrollahi followed data acquisition. Vahid Mohammadkarimi, Behnam Dalfardi, and Amir Anushiravani analyzed and interpreted the data. Mohammadkarimi and Behnam Dalfardi drafted the article. Maryam Farajzadeh, Yaser Bahramvand, Elham Nasrollahi, and Amir Anushiravani critically revised the article for important intellectual content. All authors approved the final version of the manuscript to be submitted.

Conflict of Interests: All authors declare that they have no conflict of interest.

Ethical Approval: The ethical approval code was 88/1012. Funding/Support: The Vice-Chancellor of Research at Shiraz University of Medical Sciences funded this study. 


\begin{tabular}{|c|c|c|c|c|}
\hline APACHE II score Cutoff Point & Negative Predictive Value & Positive Predictive Value & $\begin{array}{c}\text { Specificity (95\% Confidence } \\
\text { Interval) }\end{array}$ & $\begin{array}{c}\text { Sensitivity (95\% Confidence } \\
\text { Interval) }\end{array}$ \\
\hline$\leq 15$ & 66.1 & 95.2 & $96.3(89.5-99.2)$ & $60(49.7-69.7)$ \\
\hline$\leq 16$ & 67 & 93.9 & $95.1(87.8-98.6)$ & $62(51.7-71.5)$ \\
\hline$\leq 17$ & 68.5 & 90.4 & $91.4(83-96.4)$ & $66(55.8-75.2)$ \\
\hline$\leq \mathbf{1 8}$ & 75.5 & 91.6 & $91.4(83-96.4)$ & $76(66.4-84)$ \\
\hline$\leq 19$ & 76.3 & 91.7 & $91.4(83-96.4)$ & $77(67.5-84.8)$ \\
\hline$\leq \mathbf{2 0}$ & 78.4 & 87.1 & $85.2(75.5-92.1)$ & $81(71.9-88.2)$ \\
\hline$\leq \mathbf{2 1}$ & 78.6 & 84.5 & $81.5(71.3-89.2)$ & $82(73.1-89)$ \\
\hline$\leq \mathbf{2 2}$ & 79 & 83 & $79(68.5-87.3)$ & $83(74.2-89.8)$ \\
\hline$\leq \mathbf{2 3}$ & 78.5 & 81.4 & $76.5(65.8-85.2)$ & $83(74.2-89.8)$ \\
\hline$\leq \mathbf{2 4}$ & 84.5 & 80.9 & $74.1(63.1-83.2)$ & $89(81.2-94,4)$ \\
\hline$\leq \mathbf{2 5}$ & 85 & 75.2 & $63(51.5-73.4)$ & $91(83.6-95.4)$ \\
\hline
\end{tabular}

\section{References}

1. Bouch D, Thompson JP. Severity Scoring Sys Crit Ill. Continuing Education in Anaesthesia Critical Care \& Pain. 2008;8(5):181-5. doi: 10.1093/bjaceaccp/mkno33.

2. Joe BH, Jo U, Kim HS, Park CB, Hwang HJ, Sohn IS, et al. APACHE II score, rather than cardiac function, may predict poor prognosis in patients with stress-induced cardiomyopathy.J Korean Med Sci. 2012;27(1):52-7. doi: 10.3346/jkms.2012.27.1.52. [PubMed: 22219614]. [PubMed Central: PMC3247775].

3. Soleimani M, Masoudi R, Bahrami N, Qorbani M, Sadeghi T. Predicting mortality rate of patients in critical care unit using APACHE-II index. J Gorgan Univ Med Sci. 2010;11(4).

4. Mohmi H, Haghighi M. Rate of mortality in ICU patients with varying degrees. J Gilan Uni Med Sci. 2006;59:85.

5. Niewinski G, Starczewska M, Kanski A. Prognostic scoring systems for mortality in intensive care units-the APACHE model. Anaesthesiol Intensive Ther. 2014;46(1):46-9. doi: 10.5603/AIT.2014.0010. [PubMed: 24643928].
6. Cho DY, Wang YC. Comparison of the APACHE III, APACHE II and Glasgow Coma Scale in acute head injury for prediction of mortality and functional outcome. Intensive Care Med. 1997;23(1):77-84. doi: 10.1007/s001340050294. [PubMed: 9037644].

7. Giangiuliani G, Mancini A, Gui D. Validation of a severity of illness score (APACHE II) in a surgical intensive care unit. Intensive Care Med. 1989;15(8):519-22. doi: 10.1007/BF00273563. [PubMed: 2607039].

8. Donahoe L, McDonald E, Kho ME, Maclennan M, Stratford PW, Cook DJ. Increasing reliability of APACHE II scores in a medical-surgical intensive care unit: a quality improvement study. Am J Crit Care. 2009;18(1):58-64. doi: 10.4037/ajcc2009757. [PubMed: 19116406].

9. Ho KM, Lee KY, Williams T, Finn J, Knuiman M, Webb SA. Comparison of Acute Physiology and Chronic Health Evaluation (APACHE) II score with organ failure scores to predict hospital mortality. Anaesthesia.2007;62(5):466-73. doi:10.1111/j.1365-2044.2007.04999.x. [PubMed: 17448058].

10. Anushiravani A, Masoompour SM. Assessing the Performance of a Medical Intensive Care Unit: A 5-year single-center Experience. Indian J Crit Care Med.2017;21(3):163-6. doi:10.4103/ijccm.IJCCM_420_16. [PubMed: 28400688]. [PubMed Central: PMC5363106]. 